\title{
COVID-19 manifestations in people with Parkinson's disease: a USA cohort
}

\author{
Yaqian $\mathrm{Xu}^{1} \cdot$ Matthew Surface ${ }^{2} \cdot$ Amanda K. Chan $^{2} \cdot$ Joshua Halpern $^{2} \cdot$ Nora Vanegas-Arroyave $^{2,3} \cdot$ Blair Ford $^{2}$. \\ Megan P. Feeney ${ }^{4} \cdot \mathrm{Kimberly} \mathrm{T.} \mathrm{Kwei}^{2} \cdot$ Linn E. Katus ${ }^{2} \cdot$ Sheng-Han Kuo ${ }^{2,5,6} \cdot$ Hiral Shah $^{2} \cdot$ Cheryl Waters $^{2}$. \\ Linda M. Winfield ${ }^{2}$. James C. Beck ${ }^{4,7} \cdot$ Serge Przedborski ${ }^{2,5,8} \cdot$ Stanley Fahn ${ }^{2} \cdot$ Roy N. Alcalay ${ }^{2}$ (1)
}

Received: 24 April 2021 / Revised: 16 July 2021 / Accepted: 29 August 2021 / Published online: 4 September 2021

○) Springer-Verlag GmbH Germany, part of Springer Nature 2021

\begin{abstract}
Background With the explosion of COVID-19 globally, it was unclear if people with Parkinson's disease (PD) were at increased risk for severe manifestations or negative outcomes.

Objectives To report on people with PD who had suspected or confirmed COVID-19 to understand how COVID-19 manifested in PD patients.

Methods We surveyed PD patients who reported COVID-19 to their Movement Disorders specialists at Columbia University Irving Medical Center and respondents from an online survey administered by the Parkinson's Foundation that assessed COVID-19 symptoms, general clinical outcomes and changes in motor and non-motor PD symptoms.

Results Forty-six participants with PD and COVID-19 were enrolled. Similar to the general population, the manifestations of COVID-19 among people with PD were heterogeneous ranging from asymptomatic carriers (1/46) to death (6/46). The most commonly reported COVID-19 symptoms were fever/chills, fatigue, cough, weight loss, and muscle pain. Worsening and new onset of motor and non-motor PD symptoms during COVID-19 illness were also reported, including dyskinesia, rigidity, balance disturbances, anxiety, depression, and insomnia.

Conclusion We did not find sufficient evidence that PD is an independent risk factor for severe COVID-19 and death. Larger studies with controls are required to understand this further. Longitudinal follow-up of these participants will allow for observation of possible long-term effects of COVID-19 in PD patients.
\end{abstract}

Keywords Parkinson's disease · COVID-19

\section{Introduction}

The COVID-19 pandemic has affected millions of individuals around the world and there is an urgent need to identify risk factors for severe clinical outcomes. More severe

Yaqian Xu and Matthew Surface contributed equally to this work.

Roy N. Alcalay

rna2104@cumc.columbia.edu

1 Department of Psychiatry, Columbia University Irving Medical Center, New York, NY, USA

2 Department of Neurology, Columbia University Irving Medical Center, 710 West 168th St., New York, NY 10025, USA

3 Department of Neurology, Baylor College of Medicine, Houston, TX, USA
COVID-19 outcomes and a higher mortality have been described in older individuals [1] and males [2]. Since Parkinson's disease (PD) is more common in males above age 50 [3], it is imperative to determine if $\mathrm{PD}$ is a risk factor for worse COVID-19 clinical outcomes and if certain PD therapies have an effect on COVID-19 illness.

4 Parkinson's Foundation, New York, NY, USA

5 Initiative for Columbia Ataxia and Tremor, Columbia University, New York, NY, USA

6 Department of Pathology and Cell Biology, Columbia University Irving Medical Center, New York, NY, USA

7 Department of Neuroscience and Physiology, NYU Grossman School of Medicine, New York, NY, USA

8 Department of Neuroscience, Columbia University Irving Medical Center, New York, NY, USA 
Three large cohorts from Europe and the UK have published on clinical outcomes of COVID-19 in PD patients, reporting a wide range of mortality rates and potential risk factors. Fasano et al. reported a $5.7 \%$ mortality rate in a cohort of 151 PD patients with COVID-19 from a single tertiary center in Lombardy, Italy [4], and a mortality rate of $19.7 \%$ in a cohort including 117 PD patients with COVID19 from 21 tertiary centers in Italy, Iran, Spain, and the UK [5]. Sainz-Amo et al. reported the outcomes of 39 PD patients with COVID-19 from a tertiary university hospital in Spain [6]. Additionally, several smaller cohorts and case series [7-10] reported various outcomes among these patient groups. In a recent study, Vignatelli et al. [11], using a health care claims database, found that $4 / 696$ subjects with PD were admitted because of COVID-19, showing a 3-month hospitalization rate of $0.6 \%$.

When the COVID-19 pandemic reached New York, we maintained a registry of people with movement disorders who reported severe acute respiratory syndrome coronavirus 2 (SARS-CoV-2) infections or COVID-19 symptoms to their Movement Disorder specialist at Columbia University Irving Medical Center (CUIMC). In tandem with the Parkinson's Foundation (PF), we also deployed an anonymous survey to explore the emotional and behavioral consequences of the public health policies implemented to mitigate the spread of SARS-CoV-2 where respondents self-reported COVID19 status [12]. To better understand COVID-19 outcomes among people with PD, we administered a targeted telephone survey to participants with a diagnosis of PD and a confirmed or suspected diagnosis of COVID-19 from both the CUIMC registry and anonymous PF survey.

\section{Methods}

\section{Standard protocol approvals, registrations, and patient consents}

This study included questionnaire data obtained from two study protocols, both of which were approved by the CUIMC Institutional Review Board (IRB). A waiver of written documentation of consent was granted, and subjects were given a verbal presentation of the Information Sheet, followed by verbal confirmation of informed consent via telephone. The cohorts and relevant procedures of these studies are described below.

CUIMC Registry Cohort (from April 2 to Sept 16, 2020) While we do not have exact number of PD patients who contacted the clinicians, between March and May 2020, 923 formal PD-related clinic visits took place either in person or by telemedicine. CUIMC clinicians maintained a log of PD patients who reported COVID-19 or suspected COVID19. They referred family members and/or personal contacts if the patient passed away. The research team also reached out to people with PD on the CUIMC email list who completed the PF survey (detailed below) with a self-reported suspected or confirmed COVID-19.

PF Survey Cohort (from June 24 to July 9, 2020) Attendees to PF events or people who called the PF's helpline in calendar year 2019 or 2020 were invited to complete a Knowledge, Attitudes and Practice (KAP) survey via email invitation [12]. A total of 1,342 people with PD responded to the survey, and those whose survey responses reported a diagnosis of PD, a suspected or confirmed diagnosis of COVID-19, and who consented to future research were contacted for the telephone survey.

A total of 46 participant responses (39 from the CUIMC Registry Cohort and 7 from the PF Survey Cohort) were included in the pooled analyses (Online Resource-Supplementary Fig. 1).

\section{Telephone survey}

Survey questions were used or modified from the Center for Economic and Social Research-Understanding America Study- Coronavirus Tracking Survey (April 1-14, 2020) [13]. Questions for neurological manifestations of COVID19 were based on COVID-19 case series published before May 2020 [14]. Common motor and non-motor symptoms of PD were selected based on PD diagnostic criteria [15], the Unified Parkinson's Disease Rating Scale (UPDRS) and common non-motor symptoms of PD. Participants were asked to choose from one of the following categories of severity for PD symptoms during their COVID-19 illness: 1-never had the symptom; 2-new onset; 3-less severe; 4-symptom unchanged; 5-more severe. Participants who responded to the telephone survey themselves, defined as "Alive", had experienced COVID-19-related symptoms at least 2 months prior to the time of the telephone survey. The complete questionnaire is available in the Online Resource-Supplementary Survey.

\section{Statistical analyses}

Descriptive statistics were used to summarize responses. We compared the demographics and COVID-19 outcome characteristics between those alive and those who died due to COVID-19. Chi-square tests (Fisher's exact test when applicable) were used to compare categorical variables. Normality tests (Shapiro-Wilk test) were performed for continuous variables and Mann-Whitney tests were used to compare continuous variables with non-parametric distributions. Levodopa equivalent daily dosage (LEDD) was calculated as described by Tomlinson et al. [16] Statistical analysis was performed using SPSS 25.0 (SPSS Inc., Chicago, IL, USA). 


\section{Results}

Forty-six PD patients with suspected or confirmed COVID-19 (17 female/29 male) were included in the analysis. Thirty-seven had confirmed COVID-19 diagnosis by testing, including PCR, serology, or both. Mean age of the interviewed patient population was 67.9 years old (range 38-85), mean age at PD diagnosis was 60.1 years old (range 32-82), and mean PD disease duration was 7.8 years (range 0-25) (Table 1). Among those who responded to COVID-19 symptom questions, the most frequently reported symptoms were fatigue or tiredness $(N=31)$, fever or chills $(N=31)$, weight loss $(N=24)$, cough $(N=28)$, and muscle or body ache/pain $(N=14)$ (Online Resource-Supplementary Table 1). Interestingly, ten $(10 / 35,28.6 \%)$ PD patients developed temporary loss of smell and eight $(8 / 36,22.2 \%)$ PD patients developed temporary loss of taste because of COVID-19. Neurological symptoms due to COVID-19 were also reported, including weakness $(N=20)$, dizziness $(N=13)$ and confusion $(N=11)$.

COVID-19 disease severity varied among surveyed PD patients, ranging from asymptomatic to death. One PD

Table 1 Basic demographics of the study population

\begin{tabular}{|c|c|c|c|c|}
\hline & \multirow[t]{2}{*}{ Total $(N=46)$} & \multicolumn{3}{|c|}{ Alive vs. died due to COVID-19 } \\
\hline & & Alive $(N=40)$ & Died due to COVID-19 $(N=6)$ & $p^{*}$ \\
\hline Age (years old), mean (range) & $67.9(38-85)$ & $67.1(38-85)$ & $73.0(69-78)$ & 0.397 \\
\hline Age at PD diagnosis (years old), mean (range) & $60.1(32-82)$ & $60.0(32-82)$ & $60.7(46-67)$ & 0.819 \\
\hline Disease duration (years), mean (range) & $7.8(0-25)$ & $7.1(0-20)$ & $12.3(5-25)$ & 0.070 \\
\hline Gender (female), $N(\%)$ & $17(37.0 \%)$ & $16(40 \%)$ & $1(16.7 \%)$ & 0.390 \\
\hline \multicolumn{5}{|l|}{ Race/ethnicity, $N(\%)$} \\
\hline Asian & $1(2.2 \%)$ & $1(2.5 \%)$ & $0(0 \%)$ & 0.517 \\
\hline Hispanic/Latino & $8(17.4 \%)$ & $6(15.0 \%)$ & $2(33.3 \%)$ & \\
\hline White/Caucasian & $37(80.4 \%)$ & $33(82.5 \%)$ & $4(66.7 \%)$ & \\
\hline \multicolumn{5}{|l|}{ Marital status, $N(\%)$} \\
\hline Single & $2(4.3 \%)$ & $2(5.0 \%)$ & $0(0 \%)$ & 0.408 \\
\hline Married or domestic partnership & $36(78.3 \%)$ & $32(80.0 \%)$ & $4(66.7 \%)$ & \\
\hline Widowed & $2(4.3 \%)$ & $2(5.0 \%)$ & $0(0 \%)$ & \\
\hline Divorced & $6(13.0 \%)$ & $4(10.0 \%)$ & $2(33.3 \%)$ & \\
\hline \multicolumn{5}{|l|}{ Medication**, $N(\%)$} \\
\hline L-dopa & $41(89.1 \%)$ & $35(89.7 \%)$ & $6(100.0 \%)$ & 1.000 \\
\hline Amantadine & $6(13.3 \%)$ & $6(15.4 \%)$ & $0(0 \%)$ & 0.576 \\
\hline LEDD (mg), mean (range) & $392.8(0-1500.0)$ & $358.0(950.0-367.1)$ & $625.0(250.0-1500.0)$ & 0.215 \\
\hline $\begin{array}{l}\text { Duration of COVID-19 symptoms (days)**, mean } \\
\text { (range), } N(\%)\end{array}$ & $25.2(3-120)$ & $26.6(3-120)$ & $14.8(10-23)$ & 0.472 \\
\hline \multicolumn{5}{|l|}{ Hospitalized, $N(\%)$} \\
\hline Not hospitalized & $38(80.9 \%)$ & $36(90.0 \%)$ & $1(16.7 \%)$ & $<0.001$ \\
\hline Hospitalized & $7(14.9 \%)$ & $4(10.0 \%)$ & $3(50 \%)$ & \\
\hline Hospitalized and received mechanical ventilation & $2(4.3 \%)$ & $0(0 \%)$ & $2(33.3 \%)$ & \\
\hline \multicolumn{5}{|l|}{ Comorbidity**, $N(\%)$} \\
\hline Hypertension & $16(38.1 \%)$ & $13(35.1 \%)$ & $3(60 \%)$ & 0.352 \\
\hline Hypercholesterolemia & $17(38.6 \%)$ & $16(38.5 \%)$ & $1(20 \%)$ & 0.638 \\
\hline Arrhythmia/atrial fibrillation & $7(16.7 \%)$ & $6(16.2 \%)$ & $1(20 \%)$ & 1.000 \\
\hline Cancer & $7(16.7 \%)$ & $6(16.2 \%)$ & $1(20 \%)$ & 1.000 \\
\hline Renal insufficiency & $3(7.1 \%)$ & $2(5.4 \%)$ & $1(20 \%)$ & 0.323 \\
\hline Type II diabetes mellitus & $1(2.3 \%)$ & $0(0 \%)$ & $1(16.7 \%)$ & 0.140 \\
\hline
\end{tabular}

$S D$ standard deviation, $L E D D$ Levodopa equivalent daily dosage

*Fisher's exact tests were used in categorical variables, Mann-Whitney tests were used in continuous variables

**1 response was missing in Medication; 4 responses were missing in the reported duration of COVID-19 symptoms; 4 responses were missing in hypertension, arrhythmia, cancer, and renal insufficiently; 3 responses were missing in Type II diabetes mellitus; 2 responses were missing in hypercholesterolemia 
patient was asymptomatic, 18 had mild or very mild symptoms, 14 had moderate symptoms, 7 were hospitalized, and 6 died due to COVID-19. The asymptomatic patient was tested for COVID-19 after close-contact exposure and had a positive serology test result to confirm the diagnosis. All six COVID-19 deaths were in the CUIMC Registry Cohort. Of these, $83.3 \%$ (5/6) received COVID-19 testing with positive test results and were hospitalized, and two $(2 / 5,40.0 \%)$ received mechanical ventilation.

All six COVID-19 deaths were reported to have experienced fever or chills $(6 / 6,100 \%)$. Half of them $(3 / 6,50 \%)$ experienced fatigue or tiredness, cough, or confusion. Past medical history of patients who died did not differ significantly from those who survived (Table 1). Patients who died from COVID-19 had an age range of 69-78 years old, 5/6 of them were male (Online Resource-Supplementary Fig. 2). We did not find statistically significant differences in age $(p=0.397)$, gender $(p=0.390)$, ethnicity $(p=0.517)$, PD disease duration $(p=0.070)$, LEDD $(p=0.215)$, and amantadine use $(p=0.576)$ when comparing PD patients who died because of COVID-19 with those who survived due to the relatively small total number of participants in the cohort (Table 1).

PD patients also reported changes in motor and nonmotor symptoms during their COVID-19 illness. Many experienced worsening of pre-existing motor or non-motor

Table 2 Changes in Parkinson's disease motor and non-motor symptoms during COVID-19 illness

\begin{tabular}{|c|c|c|c|c|}
\hline & \multirow{2}{*}{$\begin{array}{l}\text { Those who did not have these } \\
\text { symptoms before COVID-19 } \\
\text { New onset (\%*) }\end{array}$} & \multicolumn{3}{|c|}{ Those who had these symptoms before COVID-19 } \\
\hline & & $N$ total & Less severe $(\% * *)$ & More severe $(\% * *)$ \\
\hline Bradykinesia & & 37 & & $20(54.1)$ \\
\hline Rigidity & $1(14.3)$ & 35 & $1(2.9)$ & $17(48.6)$ \\
\hline Balance & & 34 & $1(2.9)$ & $15(44.1)$ \\
\hline UPDRS-walking & $2(16.7)$ & 29 & & $13(44.8)$ \\
\hline UPDRS-mentation & $1(6.3)$ & 25 & & $12(48)$ \\
\hline UPDRS-motivation/initiative & $2(9.1)$ & 19 & & $11(57.9)$ \\
\hline OFF time & $1(6.7)$ & 23 & $1(4.3)$ & $10(43.5)$ \\
\hline UPDRS-handwriting & & 33 & $1(3)$ & $10(30.3)$ \\
\hline UPDRS-speech & & 26 & $1(3.8)$ & $10(38.5)$ \\
\hline UPDRS- dressing & $1(16.7)$ & 27 & $1(3.7)$ & $9(33.3)$ \\
\hline UPDRS-turning in bed & $3(0)$ & 23 & & $9(39.1)$ \\
\hline UPDRS-thought disorder & & 23 & & $9(39.1)$ \\
\hline Anxiety & $3(15.8)$ & 22 & $2(9.1)$ & $9(40.9)$ \\
\hline UPDRS-tremor & & 35 & $2(5.7)$ & $9(25.7)$ \\
\hline Sleep- insomnia & $2(14.3)$ & 27 & $3(11.1)$ & $8(29.6)$ \\
\hline UPDRS-hygiene & & 19 & & $7(36.8)$ \\
\hline UPDRS-swallowing & $3(4.5)$ & 15 & & $7(46.7)$ \\
\hline UPDRS-cutting food & & 19 & & $7(36.8)$ \\
\hline UPDRS-freezing & $2(7.7)$ & 16 & & $6(37.5)$ \\
\hline Constipation & $1(6.7)$ & 27 & $1(3.7)$ & $6(22.2)$ \\
\hline Pain & $1(4)$ & 15 & & $6(40.0)$ \\
\hline Urinary issues & $1(7.7)$ & 28 & & $6(21.4)$ \\
\hline Dystonia & & 17 & & $5(29.4)$ \\
\hline UPDRS-falling & & 19 & & $5(26.3)$ \\
\hline UPDRS-depression & $2(8.7)$ & 17 & & $4(23.5)$ \\
\hline UPDRS-salivation & & 15 & & $4(26.7)$ \\
\hline UPDRS-sensory symptoms & $1(6.9)$ & 17 & & $3(17.6)$ \\
\hline Dyskinesia & $2(4.3)$ & 14 & & $3(21.4)$ \\
\hline Any motor or non-motor symptom*** & $12(28.6)$ & & $8(19.0)$ & $32(76.2)$ \\
\hline
\end{tabular}

UPDRS Unified Parkinson Disease Rating Scale

*Percentage calculated out of those who did not have these symptoms before COVID-19

**Percentage calculated out of those who had these symptoms before COVID-19

****Percentage calculated out of those who responded to the symptom questions ( $N=42,4$ missing) 
symptoms during COVID-19 illness (Table 2). New onset of motor and non-motor symptoms were also reported. Interestingly, several patients reported that their motor and non-motor symptoms were less severe during the COVID19 illness, including tremor $(N=2)$, rigidity $(N=1)$, balance disturbances $(N=1)$, difficulty with handwriting $(N=1)$, problems with speech $(N=1)$, problems with OFF time $(N=1)$, insomnia $(N=3)$, and anxiety $(N=2)$.

\section{Discussion}

In this study, we analyzed a cohort of PD patients from the USA with suspected or confirmed COVID-19. Our results showed that the presentation of COVID-19 was heterogeneous among PD patients with varied severity and outcomes, ranging from asymptomatic (1/46) to fatal (6/46). Our cohort included 46 PD patients with COVID-19. This is a relatively large cohort of PD COVID-19 patients and the only cohort from the USA. Similar to what the European and the UK cohorts reported [4-6], COVID-19 severity varied among PD patients with no single factor determining the outcome of COVID-19 in patients with PD.

Similar to the general population, the most common COVID-19 symptoms included fever or chills, fatigue, cough, weight loss and muscle pain. PD patients also frequently reported worsened motor (e.g., bradykinesia in 54\% of patients, rigidity in $49 \%$ of patients, and balance disturbances in $44 \%$ of patients) and non-motor symptoms (e.g., problems with motivation in $58 \%$ of patients, and intellectual impairment in $48 \%$ of patients) during their COVID-19 illness, as well as a few cases of new onset motor symptoms and non-motor symptoms. Interestingly, a few participants reported improved PD symptoms during COVID-19 illness, including tremor, rigidity, balance disturbances, handwriting, speech, OFF time, insomnia, and anxiety. To our knowledge, this phenomenon has yet to be reported. Since formal examination before and after COVID-19 is not available and a control group is lacking, this observation should be viewed with caution. Future studies including a control group will help us understand the association between symptom changes and COVID-19 in PD patients.

PD medication has been a major focal point in the prediction of COVID-19 severity. A Spanish cohort [6] reported that a lower frequency of dopamine agonists use was associated with severe COVID-19: 5 out of 29 PD patients with severe COVID-19 were taking dopamine agonists comparing with 74 out of 182 PD patients with mild COVID-19 or no COVID-19 symptoms. Our study did not show a trend in dopamine agonists use when comparing PD patients who died from COVID-19 with those who survived. However, we observed that all six patients (6/40) taking amantadine at the time of COVID-19 illness survived. Of note, a case series from Poland reported five PD patients who tested positive for SARS-CoV-2 while taking amantadine, and none of them developed clinical manifestation of infectious disease [10]. These observations are intriguing because amantadine has antiviral properties against strains of influenza A. These findings may support amantadine's hypothesized biological efficacy in COVID-19 treatment [17-20]. Therefore, PD medications, like dopamine agonists, may play a role in mitigating the risk of severe COVID-19 illness in PD, but the true effect of these medications and the underlying mechanism(s) require further exploration.

The major limitation of our study was the referral system. We neither actively contacted all PD patients treated at CUIMC nor tested them for SARS-CoV-2 via PCR or serology. Patients and family members are more likely to contact CUIMC clinicians for severe disease or death than for a milder disease course, and relying on patients' and family members' report to the clinicians may be biased towards identifying more severe cases. Conversely, relying on email questionnaires likely biases us towards milder COVID-19 cases in patients who were able to complete a questionnaire upon recovery. In the CUIMC cohort, we observed a casefatality rate of $15.4 \%(6 / 39)$, which is higher than the $5.7 \%$ mortality rate (6/105) in PD patients from a communitybased case-control study in Lombardy, Italy [4], but lower than both a multicenter cohort from Europe and the UK [5] and a case series of ten cases from Italy and the UK [7] which reported $19.7 \%$ and $40 \%$ mortality, respectively. While the relatively low number of fatalities is informative and reassuring that most people with PD and COVID-19 recover, none of the statistical analyses comparing survivors to non-survivors reached significance, likely because of the small sample size of fatality cases. Additionally, our cohort was skewed towards White/Caucasian (80.4\%). Furthermore, only $0.4 \%$ (6/1342) of the participants who responded to the PF online KAP survey self-reported African-American ancestry [12]. Further studies examining more racially diverse cohorts are needed to determine the effects of COVID-19 in PD patients from underrepresented groups. Currently, there is a study underway at CUIMC seeking to understand the ability to adhere to social distancing guidelines as well as COVID-19 outcomes in patients with neurological disorders in Upper Manhattan and the Bronx, which may help us understand the impact of COVID-19 in a local, racially diverse PD cohort.

Much remains unknown about the effect of COVID-19 on people with PD. Our results showed the presentation of COVID-19 was heterogeneous among PD patients, with varied perceived severity and clinical outcomes. Results from this study align with previous findings, including new and worsened PD symptoms in PD patients with COVID19 [4, 8, 21-23], while also presenting an intriguing novel observation of PD symptom improvement during COVID-19 
illness. Further studies, including longitudinal follow-up, are required to further elucidate the relationship between $\mathrm{PD}$ and COVID-19.

Supplementary Information The online version contains supplementary material available at https://doi.org/10.1007/s00415-021-10784-3.

Acknowledgements We thank those living with PD and their families for participating in this study.

Author contributions Conceptualization and methodology: All authors contributed to the study conception and design; formal analysis and investigation: YX, MS, RNA; writing — original draft preparation: YX, MS; writing - review and editing: AKC, JH, NV-A, BF, MPF, KTK, LEK, S-HK, HS, CW, LMW, JCB, SP, SF, RNA; resources: AKC, JH, NV-A, BF, MPF, KTK, LEK, S-HK, HS, CW, LMW, JCB, SP, SF, RNA; supervision: RNA.

Funding No funding was received for conducting this study.

Availability of data and material Data and relevant material can be shared upon reasonable request to the corresponding author.

Code availability Analyses in this study were performed using SPSS 25.0 (SPSS Inc., Chicago, IL, USA), SPSS syntax can be shared upon reasonable request.

\section{Declarations}

Conflicts of interest The authors have no relevant financial or nonfinancial interests to disclose.

Ethical approval The questionnaire and methodology for this study was approved by the Human Research Ethics committee of the Columbia University Irving Medical Center (Ethics approval number: AAAT1585, AAAT0369).

Consent to participate A waiver of written documentation of consent was granted, and subjects were given a verbal presentation of the Information Sheet, followed by verbal confirmation of informed consent via telephone.

Consent for publication Not applicable. No identifying information was reported.

\section{References}

1. Shahid Z, Kalayanamitra R, McClafferty B, Kepko D, Ramgobin D, Patel R, Aggarwal CS, Vunnam R, Sahu N, Bhatt D, Jones K, Golamari R, Jain R (2020) COVID-19 and older adults: what we know. J Am Geriatr Soc 68:926-929

2. Garg S (2020) Hospitalization rates and characteristics of patients hospitalized with laboratory-confirmed coronavirus disease 2019-COVID-NET, 14 States, March 1-30, 2020. MMWR Morb Mortal Wkly Rep 69:458-464

3. Van Den Eeden SK, Tanner CM, Bernstein AL, Fross RD, Leimpeter A, Bloch DA, Nelson LM (2003) Incidence of Parkinson's disease: variation by age, gender, and race/ethnicity. Am J Epidemiol 157:1015-1022
4. Fasano A, Cereda E, Barichella M, Cassani E, Ferri V, Zecchinelli AL, Pezzoli G (2020) COVID-19 in Parkinson's disease patients living in Lombardy, Italy. Mov Disord 35:1089-1093

5. Fasano A, Elia AE, Dallocchio C, Canesi M, Alimonti D, Sorbera C, Alonso-Canovas A, Pezzoli G (2020) Predictors of COVID-19 outcome in Parkinson's disease. Park Relat Disord 78:134-137

6. Sainz-Amo R, Baena-Álvarez B, Pareés I, Sánchez-Díez G, PérezTorre P, López-Sendón JL, Fanjul-Arbos S, Monreal E, CorralCorral I, García-Barragán N, Martínez-Castrillo JC, Fasano A, Alonso-Cánovas A (2020) COVID-19 in Parkinson's disease: what holds the key? J Neurol 268(8):2666-2670

7. Antonini A, Leta V, Teo J, Chaudhuri KR (2020) Outcome of Parkinson's disease patients affected by COVID-19. Mov Disord 35:905-908

8. Cilia R, Bonvegna S, Straccia G, Andreasi NG, Elia AE, Romito LM, Devigili G, Cereda E, Eleopra R (2020) Effects of COVID19 on Parkinson's disease clinical features: a community-based case-control study. Mov Disord 35:1287-1292

9. Hainque E, Grabli D (2020) Rapid worsening in Parkinson's disease may hide COVID-19 infection. Park Relat Disord $75: 126-127$

10. Rejdak K, Grieb P (2020) Adamantanes might be protective from COVID-19 in patients with neurological diseases: multiple sclerosis, Parkinsonism and cognitive impairment. Mult Scler Relat Disord 42:102163

11. Vignatelli L, Zenesini C, Belotti LMB, Baldin E, Bonavina G, Calandra-Buonaura G, Cortelli P, Descovich C, Fabbri G, Giannini G, Guarino M, Pantieri R, Samoggia G, Scaglione C, Trombetti S, D’Alessandro R, Nonino F (2021) Risk of hospitalization and death for COVID-19 in people with Parkinson's disease or Parkinsonism. Mov Disord 36:1-10

12. Feeney MP, Xu Y, Surface M, Shah H, Vanegas-Arroyave N, Chan AK, Delaney E, Przedborski S, Beck JC, Alcalay RN (2021) The impact of COVID-19 and social distancing on people with Parkinson's disease: a survey study. NPJ Park Dis 7:10

13. Toolkit P (2020) COVID-19 protocols. https://www.phenxtoolk it.org/covid19/source. Accessed 30 Sept 2020

14. Mao L, Jin H, Wang M, Hu Y, Chen S, He Q, Chang J, Hong C, Zhou Y, Wang D, Miao X, Li Y, Hu B (2020) Neurologic manifestations of hospitalized patients with coronavirus disease 2019 in Wuhan, China. JAMA Neurol 77:683-690

15. Postuma RB, Berg D, Stern M, Poewe W, Olanow CW, Oertel W, Obeso J, Marek K, Litvan I, Lang AE, Halliday G, Goetz CG, Gasser T, Dubois B, Chan P, Bloem BR, Adler CH, Deuschl G (2015) MDS clinical diagnostic criteria for Parkinson's disease. Mov Disord 30:1591-1601

16. Tomlinson CL, Stowe R, Patel S, Rick C, Gray R, Clarke CE (2010) Systematic review of levodopa dose equivalency reporting in Parkinson's disease. Mov Disord 25:2649-2653

17. Abreu GEA, Aguilar MEH, Covarrubias DH, Durán FR (2020) Amantadine as a drug to mitigate the effects of COVID-19. Med Hypotheses 140:109755

18. Aranda-Abreu GE, Aranda-Martínez JD, Araújo R (2021) Use of amantadine in a patient with SARS-CoV-2. J Med Virol 93(1):110-111

19. Aranda-Abreu GE, Aranda-Martínez JD, Araújo R, HernándezAguilar ME, Herrera-Covarrubias D, Rojas-Durán F (2020) Observational study of people infected with SARS-Cov-2, treated with amantadine. Pharmacol Rep 73(6):1538-1541

20. Smieszek SP, Przychodzen BP, Polymeropoulos MH (2020) Amantadine disrupts lysosomal gene expression: a hypothesis for COVID19 treatment. Int J Antimicrob Agents 55:106004

21. Brown EG, Chahine LM, Goldman SM, Korell M, Mann E, Kinel DR, Arnedo V, Marek KL, Tanner CM (2020) The effect of the COVID-19 pandemic on people with Parkinson's disease. J Park Dis 10(4):1365-1377 
22. de Marcaida JA, Lahrmann J, Machado D, Bluth L, Dagostine M, Moro-de Casillas M, Bortan E, Kanchana S, Alberts M (2020) Clinical characteristics of coronavirus disease 2019 (COVID-19) among patients at a movement disorders center. Geriatrics (Basel) $5: 54$
23. Li J, Long X, Zhu C, Wang R, Hu S, Wang T, Li J, Lin Z, Xiong N (2020) Management of a Parkinson's disease patient with severe COVID-19 pneumonia. Ther Adv Chronic Dis 11:2040622320949423 